\title{
A finite element method for poro mechanical modelling of geotechnical problems using local second gradient models
}

\author{
F. Collin ${ }^{1, \ddagger}$, R. Chambon ${ }^{2, *, \dagger}$ and R. Charlier ${ }^{3}$ \\ ${ }^{1}$ ULg, Geomac, Belgium \\ ${ }^{2}$ Laboratoire $3 S$, UJF-INPG-CNRS, Grenoble, France \\ ${ }^{3}$ Université de Liège, Geomac, Belgium
}

\begin{abstract}
SUMMARY
In this paper, a new finite element method is described and applied. It is based on a theory developed to model poromechanical problems where the mechanical part is obeying a second gradient theory. The aim of such a work is to properly model the post localized behaviour of soils and rocks saturated with a pore fluid. Beside the development of this new coupled theory, a corresponding finite element method has been developed. The elements used are based on a weak form of the relation between the deformation gradient and the second gradient, using a field of Lagrange multipliers. The global problem is solved by a system of equations where the kinematic variables are fully coupled with the pore pressure. Some numerical experiments showing the effectiveness of the method ends the paper. Copyright (C) 2005 John Wiley \& Sons, Ltd.
\end{abstract}

KEY WORDS: finite element; Lagrange multipliers; second gradient models; localization; post localization; poromechanics; hydromechanics

\section{INTRODUCTION}

Soils and rocks are generally considered as porous materials filled by one or several fluids. Interactions between the porous skeleton and the fluid phases are very important in many engineering problems. It is therefore necessary to study applications involving multiphasic materials. Different approaches have been proposed in the past to tackle such kind of problems. The first one is the general theory of porous media (based on a mixture theory) which could be

\footnotetext{
*Correspondence to: R. Chambon, Laboratoire 3S, Grenoble Université Joseph Fourier, Institut National Polytechnique, C.N.R.S. U.M.R. 5521, B.P. 53X, 38041 Grenoble Cedex, France.

†E-mail: rene.chambon@hmg.inpg.fr

*FNRS Post-doctoral Researcher.
}

Contract/grant sponsor: Fonds National de la Recherche Scientifique, Belgium

Contract/grant sponsor: European project RTN-DIGA; contract/grant number: HPRN-CT-2002-00220

Received 6 January 2005

Revised 13 July 2005

Copyright (c) 2005 John Wiley \& Sons, Ltd.

Accepted 17 August 2005 
referred to the works of Bowen [1] and de Boer [2]. Using an averaging theory [3,4], Lewis and Schrefler [5] propose the general equation for multiphasic media. Finally, a third approach follows the ideas of Biot [6] in the framework of thermodynamics of irreversible processes. This has been developed by Coussy [7]. All these theories are only based on simple materials as defined by Truesdell and Noll [8].

When submitted to large strains, geomaterials exhibit clear localized ruptures (like shear bands $[9,10]$, or compaction bands $[11,12])$, this phenomenon has been extensively studied, especially when the geomaterial is assumed to be a monophasic material. In this case, numerical modelling of such kind of localized shear zones suffers of pathological sensitivity to the finite element discretization. One of the well known consequences is that rupture can occur without energy consumption [13] which is physically non-admissible. The reason of such a behaviour has now been clarified. It has been proved that an enhanced model incorporated an internal length is necessary to provide an objective description of post localized loading paths. Many enhancements have been proposed and studied in the past since the pioneering work of Aifantis [14] (see Reference [15]). The non-local approach was first proposed for damage concrete mechanics [16] and extended later to other materials. Another type of enhancement corresponds to constitutive models with gradient of internal variables or with non-local internal variables $[17,15]$. A last family of enriched models comes from continua with microstructure. This is the case for Cosserat models [18] (see Reference [19] or [15] for applications to geomaterials) or for local second gradient ones [20-23] (see References [24, 25] for application to geomaterials). It is not the aim of this paper to compare the advantages and the shortcomings of every class of enhanced models. Readers interested by such a discussion can have a look to the references of our paper co-authored with Caillerie and Tamagnini [26].

In the framework of biphasic porous media, there is very few constitutive models introducing an internal length-scale. The most important works made in this topic have been performed by Ehlers and coworkers who used Cosserat continuum in coupled problems within a large strain elasto plastic framework [27,28]. A gradient-dependent plasticity model has been recently extended in two and three phases porous media by Zhang and Schefler [29].

In fact, the pore fluid equation seems introducing its own internal scale, which competes with the length-scale introduced by enhanced models. Stability analysis, in the restricted case of the so called linear comparison solid, have been performed to evaluate the relative importance of each scale [30] and the instability conditions [31].

In the present paper, the purpose is to develop a coupled local second gradient model in order to study hydromechanical applications. After a section describing the main notations, a quasi-static formulation (which means that all inertia terms are neglected) of a coupled microstructured medium is proposed. The analysis is restricted to a porous skeleton filled by one compressible fluid. In order to solve numerically the field equations, a linear auxiliary problem is defined following the ideas of Borja [32] (Section 5). In all the developments, the geometrical effects are taken into account, which means that for the skeleton the current configuration is different from the reference one. In Section 6, the linear auxiliary problem is discretized using the finite element methodology [33]. A large strain isoparametric coupled finite element is proposed. It should be noted that geometrical effects introduce additional coupling terms in the formulation. In the following section, a plane strain compression test is modelled. For the sake of simplicity, the constitutive equation of the skeleton is an hypo elasto-plastic model. Developments towards a hyper elasto-plastic one belonging to the framework defined in Reference [26] has to be made. The preliminary numerical 
computations presented at the end of the paper show clearly the regularization of the localization pattern.

\section{NOTATIONS}

A component is denoted by the name of the tensor (or vector) accompanied with tensorial indices. All tensorial indices are in lower position as there is no need in the following of a distinction between covariant and contravariant components. The summation convention with respect to repeated tensorial indices is adopted throughout.

The time $t$ plays an important role in the following, it is always indicated as an index in the upper position. Derivation with respect to time is denoted by a dot. These time derivative are material time derivatives, this means that they are computed for the same material (skeleton) mass.

All the equations are written in the current (or spatial) configuration of the skeleton at a given time $t$. All the functions defined in the following can be seen as functions of the reference position of the skeleton denoted $x_{i}^{0}$. Since the current configuration is known, the mappings $x_{i}^{t}\left(x_{i}^{0}\right)$ and $x_{i}^{0}\left(x_{i}^{t}\right)$ between this configuration and the reference one are known. Finally, all the functions can be seen as function of $x_{i}^{0}$ or $x_{i}^{t}$. In order to simplify the notations we use the same notation for the functions and theirs values. So we will write for any function $a: a\left(x_{i}^{0}\right)=a\left(x_{i}^{0}\left(x_{i}^{t}\right)\right)=a\left(x_{i}^{t}\right)$.

Stresses are defined following the classical continuum mechanics conventions. Consequently, positive pore pressures are in fact negative isotropic stresses.

\section{BALANCE EQUATIONS OF A MICROSTRUCTURED POROUS MEDIUM}

Geomaterials like soils, rocks and concrete are porous media generally considered as the superposition of two continua [7]: the solid skeleton (grains assembly) and the fluid phases (water, air, oil,...). For this study, our analysis is restricted to saturated conditions. It is assumed that there are two immiscible phases: the porous skeleton and one fluid (water in the usual case). Phase changes like evaporation and dissolution are not considered. Moreover solid grains are assumed here to be incompressible.

\subsection{Classical poromechanics}

Based on averaging theories [3,4], Lewis and Schrefler [5] proposed the governing equations for the full dynamic behaviour of a partially saturated porous medium. Hereafter, these equations are restricted for quasi-static problem in saturated conditions. In the following developments, the balance equations of the coupled problem are written in the current solid configuration denoted $\Omega^{t}$ (updated Lagrangian formulation). Large strain effects are taken into account and their contribution in the formulation can be clearly described (see Reference [34] for more details).

The balance equations can be written separately for both phases or alternatively for the whole mixture on one hand and one of the two phases on the other hand. All along this paper, this second way is used. 


\subsubsection{Balance of momentum}

Balance of momentum for the mixture: In the mixture balance of momentum equation, the interaction forces between fluid phase and grain skeleton cancelled. In a weak form (virtual work principle), this equation reads: for any kinematically admissible virtual displacement field $u_{i}^{\star}$ :

$$
\int_{\Omega^{t}} \sigma_{i j}^{t} \varepsilon_{i j}^{\star} \mathrm{d} \Omega^{t}=\int_{\Omega^{t}}\left(\varrho^{s}\left(1-\phi^{t}\right)+\varrho^{w, t} \phi^{t}\right) g_{i} u_{i}^{\star} \mathrm{d} \Omega^{t}+\int_{\Gamma_{\sigma}^{t}} \bar{t}_{i}^{t} u_{i}^{\star} \mathrm{d} \Gamma^{t}
$$

where $\varepsilon_{i j}^{\star}=\frac{1}{2}\left(\left(\partial u_{i}^{\star} / \partial x_{j}^{t}\right)+\left(\partial u_{j}^{\star} / \partial x_{i}^{t}\right)\right)$ is the kinematically admissible virtual strain field, $\phi^{t}$ is the porosity defined as $\phi^{t}=\Omega^{w, t} / \Omega^{t}$ where $\Omega^{t}$ is the current volume of a given mass of skeleton and $\Omega^{w, t}$ the corresponding volume of fluid, $\varrho^{s}$ is the solid grain density, $\varrho^{w, t}$ is the fluid density, $g_{i}$ is the gravity acceleration.

Grains are assumed to be incompressible which means $\varrho^{s}=$ cte and explains why there is no superscript $t$ for the grain density. The first right-hand term of Equation (1) corresponds to gravity volume force of the mixture. $\Gamma_{\sigma}^{t}$ is the part of the boundary where tractions $\bar{t}_{i}^{t}$ are known.

Balance of momentum for the fluid phase: It is written in a strong form

$$
\frac{\partial p^{t}}{\partial x_{i}^{t}}+F_{i}^{S / W, t}+\varrho^{w, t} g_{i}=0
$$

where $\phi^{t}$ has been cancelled. In fact $\phi^{t} F_{i}^{S / W, t}$ is the true drag force applied on to the fluid by the skeleton, $p$ is the pore pressure.

3.1.2. Mass balance equations. Mass balance equations are written on one hand for the solid and on the other hand for the fluid. The first one is written in strong form whereas the second one is written in a weak form.

Mass balance equation for the solid phase: Since the current configuration used is defined following the skeleton movement, the mass balance equation of the solid skeleton is necessarily met. For a given mixture volume $\Omega^{t}$, mass balance equation reads

$$
\frac{\partial\left(\varrho^{s}\left(1-\phi^{t}\right) \Omega^{t}\right)}{\partial t}=0
$$

Mass balance equation for the fluid: In a weak form, this equation reads

$$
\int_{\Omega^{t}}\left(\dot{M}^{t} p^{\star}-m_{i}^{t} \frac{\partial p^{\star}}{\partial x_{i}^{t}}\right) \mathrm{d} \Omega^{t}=\int_{\Omega^{t}} Q^{t} p^{\star} \mathrm{d} \Omega^{t}-\int_{\Gamma_{q}^{t}} \bar{q}^{t} p^{\star} \mathrm{d} \Gamma^{t}
$$

$Q^{t}$ is a sink term and $\Gamma_{q}^{t}$ is the part of the boundary where the input fluid mass per unit area $\bar{q}^{t}$ is prescribed. $M^{t}$ is the mass of the fluid phase inside the current configuration of the skeleton $\Omega^{t}$ defined hereafter in Equation (11). The mass flow $m_{i}^{t}$ is defined as follows:

$$
m_{i}^{t}=\varrho^{w, t} \phi^{t} V_{i}^{W / S, t}
$$

where $V_{i}^{W / S, t}$ is the true fluid velocity with respect to the solid phase. 
3.1.3. Constitutive equations. In order to solve the problem described in the previous sections it is necessary to add the constitutive equations.

The first one gives the total stress $\sigma_{i j}^{t}$ as a function of the kinematics. Here we assume first that the Terzaghi's definition of effective stress holds:

$$
\sigma_{i j}^{t}=\sigma_{i j}^{\prime t}-p^{t} \delta_{i j}
$$

Then the effective stress $\sigma_{i j}^{\prime t}$ is assumed to be related to the history of the kinematics of the skeleton using any usual monophasic constitutive equation.

Second we assume that the drag forces are given by the law:

$$
F_{i}^{S / W, t}=\frac{\varrho^{w, t} \phi^{t} g}{K} V_{i}^{W / S, t}
$$

$F_{i}^{S / W, t}$ is the true drag force applied on the fluid by the skeleton for a current unit volume of the mixture divided by the porosity. $K$ is the Darcy permeability $(\mathrm{m} / \mathrm{s})$ and $g$ is the norm of $g_{i}$.

Third the compressible fluid is assumed to respect the following relationship [5]. This predicts an increase of fluid density as a function of the pore pressure, defining $k^{w}$ as the fluid bulk modulus:

$$
\dot{\varrho}^{w, t}=\frac{\varrho^{w, t}}{k^{w}} \dot{p}^{t}
$$

and finally the grains are assumed to be incompressible which means $\rho^{s}=$ cte.

3.1.4. Computation of the mass flow and the variation of fluid mass. The mass flow $m_{i}^{t}$ is defined in Equation (5) and is obtained using the Darcy's law (Equation (7)) and the balance of momentum for the fluid (Equation (2)) as follows:

$$
m_{i}^{t}=-\frac{K}{g}\left(\frac{\partial p^{t}}{\partial x_{i}^{t}}+\varrho^{w, t} g_{i}\right)=-\varrho^{w, t} \frac{\kappa}{\mu}\left(\frac{\partial p^{t}}{\partial x_{i}^{t}}+\varrho^{w, t} g_{i}\right)
$$

where $\kappa$ is the intrinsic permeability and $\mu$ is the fluid viscosity.

In a volume $\Omega^{t}$, the fluid mass is equal to

$$
M_{\Omega^{t}}^{t}=\varrho^{w, t} \phi^{t} \Omega^{t}
$$

Variations of fluid mass content are related to the compressibility of the fluid phase and the skeleton (even if the grains themselves are incompressible). The time derivative of this fluid mass is obtained directly by using Equations (8) and (3), this yields for a unit mixture volume:

$$
\dot{M}^{t}=\varrho^{w, t}\left[\frac{\dot{p}^{t}}{k^{w}} \phi^{t}+\frac{\dot{\Omega}^{t}}{\Omega^{t}}\right]
$$

3.1.5. Boundary conditions. In order to get a well posed problem it is necessary finally to detail the boundary conditions.

For any point belonging to $\Gamma_{\sigma}^{t}$, which normal is denoted $n_{j}^{t}$ :

$$
\sigma_{i j}^{t} n_{j}^{t}=\bar{t}_{i}^{t}
$$

where $\bar{t}_{i}^{t}$ is given. On the other hand the displacements are known on the remain of $\Gamma^{t}$. 
Similarly, for any point belonging to $\Gamma_{q}^{t}$ :

$$
\bar{q}^{t}=m_{i}^{t} n_{i}^{t}
$$

where $\bar{q}^{t}$ is given. On the other hand the pressure is known on the remain of $\Gamma^{t}$.

\subsection{Local second gradient model for monophasic medium}

Let us recall first the governing equations of a monophasic medium with microstructure. This kind of models can be traced back to the pioneering work of the Cosserat brothers [18], Mindlin [22] and Germain [20]. In the framework of microstructure continuum theory, a microkinematic gradient field $v_{i j}$ is introduced to describe strain and rotation at the microscale. With respect to classical continuum mechanics, additional terms are then added in the internal virtual work of a given body [20]. The following expression holds for any virtual quantities:

$$
W_{i}^{*}=\int_{\Omega^{t}}\left(\sigma_{i j}^{t} \frac{\partial u_{i}^{*}}{\partial x_{j}^{t}}+\tau_{i j}^{t}\left(v_{i j}^{*}-F_{i j}^{*}\right)+\Sigma_{i j k}^{t} h_{i j k}^{*}\right) \mathrm{d} \Omega^{t}
$$

where $F_{i j}^{*}$ is the virtual macrodeformation gradient, $v_{i j}^{*}$ is the virtual microkinematic gradient, $\tau_{i j}^{t}$ called microstress is an additive stress associated to the microstructure, $h_{i j k}^{*}$ is the virtual microsecond gradient and $\Sigma_{i j k}^{t}$ is the double stress dual of $h_{i j k}^{*}$.

In the local second gradient model used in the following, an assumption is added: the micro kinematic gradient is equal to the macrodeformation gradient

$$
v_{i j}=F_{i j}
$$

As a consequence: $v_{i j}^{*}=F_{i j}^{*}$. Finally, the principle of virtual work can be rewritten as follows:

$$
\int_{\Omega^{t}}\left(\sigma_{i j}^{t} \frac{\partial u_{i}^{*}}{\partial x_{j}^{t}}+\Sigma_{i j k}^{t} \frac{\partial^{2} u_{i}^{*}}{\partial x_{j}^{t} \partial x_{k}^{t}}\right) \mathrm{d} \Omega^{t}=W_{e}^{*}
$$

Assuming that the boundary $\Omega^{t}$ is regular, the external virtual work $W_{e}^{*}$ can be defined as follows:

$$
W_{e}^{*}=\int_{\Omega^{t}} \varrho g_{i} u_{i}^{*} \mathrm{~d} \Omega_{\sigma}^{t}+\int_{\Gamma_{\sigma}^{t}}\left(\bar{t}_{i} u_{i}^{*}+\bar{T}_{i} D u_{i}^{*}\right) \mathrm{d} \Gamma^{t}
$$

where $\bar{t}_{i}$ is the external (classical) forces per unit area and $\bar{T}_{i}$ an additional external (double) force per unit area, both applied on a part $\Gamma_{\sigma}^{t}$ of the boundary of $\Omega^{t}$. D denotes the normal derivative of any quantity $q,\left(D q=\left(\partial q / \partial x_{k}\right) n_{k}\right)$. To the authors' knowledge, the additional boundary condition (on $\bar{T}_{i}$ ) allows to produce solutions with boundary layers. In this work, our interest is focused only on strain localization. This is why we have assumed that the double forces are equal to zero.

Such a local approach has been developed by Chambon et al. [25, 35, 36] for geomaterials and by Fleck and Hutchinson [37] for metals. One of the advantages of local second gradient is the fact that constitutive equations remain local and therefore it is straightforward to formulate 
a second gradient extension of any classical models. For instance it is possible to develop a hyperelastoplastic constitutive equation as it has been made by Chambon et al. [26].

The previous virtual work equation of second gradient models can of course be used in a finite element code. However, this equation needs the use of $C^{1}$ functions for the displacement field as second derivatives of the displacement are involved. In order to avoid such functions in the virtual work principle (16), the equalities between $v_{i j}^{*}$ and $F_{i j}^{*}$ and between $v_{i j}$ and $F_{i j}$ are introduced through a field of Lagrange multipliers $\lambda_{i j}$ related to a weak form of the constraint (15) (see Reference [24]):

$$
\begin{array}{r}
\int_{\Omega^{t}}\left(\sigma_{i j}^{t} \frac{\partial u_{i}^{*}}{\partial x_{j}^{t}}+\Sigma_{i j k}^{t} \frac{\partial v_{i j}^{*}}{\partial x_{k}^{t}}\right) \mathrm{d} \Omega^{t}-\int_{\Omega^{t}} \lambda_{i j}^{t}\left(\frac{\partial u_{i}^{*}}{\partial x_{j}^{t}}-v_{i j}^{*}\right) \mathrm{d} \Omega^{t}-W_{e}^{*}=0 \\
\int_{\Omega^{t}} \lambda_{i j}^{*}\left(\frac{\partial u_{i}}{\partial x_{j}^{t}}-v_{i j}^{t}\right) \mathrm{d} \Omega^{t}=0
\end{array}
$$

\subsection{Local second gradient coupled model}

Starting from Equations (1) and (4) of the coupled problem in classical poromechanics, microstructure effects can be introduced in the momentum balance equation by adding microkinematic gradient terms, under the previous assumptions $\left(v_{i j}^{*}=F_{i j}^{*}, v_{i j}=F_{i j}\right)$. Assuming that pore fluid has no influence at the microstructure level, microkinematic gradient are not generated by pore pressure variations. This latter hypothesis follows the ideas of Ehlers [27] concerning a Cosserat model for biphasic medium. Cosserat model [18] is in fact a particular case of microstructured medium [24].

According to the previous assumptions, we have the following governing equations:

$$
\begin{aligned}
\int_{\Omega^{t}}\left(\sigma_{i j}^{t} \frac{\partial u_{i}^{*}}{\partial x_{j}^{t}}+\Sigma_{i j k}^{t} \frac{\partial^{2} u_{i}^{*}}{\partial x_{j}^{t} \partial x_{k}^{t}}\right) \mathrm{d} \Omega^{t}=\int_{\Omega^{t}} \varrho^{\mathrm{mix}, t} g_{i} u_{i}^{*} \mathrm{~d} \Omega^{t}+\int_{\Gamma_{\sigma}^{t}}\left(\bar{t}_{i} u_{i}^{*}+\bar{T}_{i} D u_{i}^{*}\right) \mathrm{d} \Gamma^{t} \\
\int_{\Omega^{t}}\left(\dot{M}^{t} p^{\star}-m_{i}^{t} \frac{\partial p^{\star}}{\partial x_{i}^{t}}\right) \mathrm{d} \Omega^{t}=\int_{\Omega^{t}} Q^{t} p^{\star} \mathrm{d} \Omega^{t}-\int_{\Gamma_{q}^{t}} \bar{q}^{t} p^{\star} \mathrm{d} \Gamma^{t}
\end{aligned}
$$

where $\varrho^{\text {mix }, t}$ is the mass density of the mixture $\left(\varrho^{\text {mix }, t}=\varrho^{s}\left(1-\phi^{t}\right)+\varrho^{w, t} \phi^{t}\right)$. Introducing Lagrange multiplier fields (like in the previous section), the governing equations of the local second gradient coupled problem are then the following:

$$
\begin{aligned}
& \int_{\Omega^{t}}\left(\sigma_{i j}^{t} \frac{\partial u_{i}^{*}}{\partial x_{j}^{t}}+\Sigma_{i j k}^{t} \frac{\partial v_{i j}^{*}}{\partial x_{k}^{t}}\right) \mathrm{d} \Omega^{t}-\int_{\Omega^{t}} \lambda_{i j}^{t}\left(\frac{\partial u_{i}^{*}}{\partial x_{j}^{t}}-v_{i j}^{*}\right) \mathrm{d} \Omega^{t} \\
& =\int_{\Omega^{t}}\left(\varrho^{s}\left(1-\phi^{t}\right)+\varrho^{w, t} \phi^{t}\right) g_{i} u_{i}^{*} \mathrm{~d} \Omega^{t}+\int_{\Gamma_{\sigma}^{t}}\left(\bar{t}_{i} u_{i}^{*}+\bar{T}_{i} v_{i k}^{*} n_{k}\right) \mathrm{d} \Gamma^{t}
\end{aligned}
$$




$$
\begin{aligned}
\int_{\Omega^{t}} \lambda_{i j}^{*}\left(\frac{\partial u_{i}}{\partial x_{j}^{t}}-v_{i j}^{t}\right) \mathrm{d} \Omega^{t} & =0 \\
\int_{\Omega^{t}}\left(\dot{M}^{t} p^{\star}-m_{i}^{t} \frac{\partial p^{\star}}{\partial x_{i}^{t}}\right) \mathrm{d} \Omega^{t} & =\int_{\Omega^{t}} Q^{t} p^{\star} \mathrm{d} \Omega^{t}-\int_{\Gamma_{q}^{t}} \bar{q}^{t} p^{\star} \mathrm{d} \Gamma^{t}
\end{aligned}
$$

In the present work, the stress tensor $\sigma_{i j}$ in Equation (22) is a total stress defined according to Terzaghi's postulate (6), on the contrary the double stress $\Sigma_{i j k}$ has no link with the pore pressure.

Equations (22)-(24) have to hold for any time $t$, the virtual quantities in these equations being dependant on the history of boundary conditions and then on time $t$. Moreover the constitutive equations also have to hold for any time $t$.

\section{TIME DISCRETIZATION}

Considering a body submitted to a given loading path driven by the boundary conditions history, solving the problem is finding the unknown fields $u_{i}, v_{i j}, \lambda_{i j}$ and $p$, for which equations from (22) to (24) hold, the skeleton constitutive equation and the boundary conditions for any time $t$. In order to solve numerically this non-linear problem, the loading process is discretized into finite time steps $\Delta t$. The system of equations is then solved for any time $t$ belonging to a sequence like: $0, \Delta t, 2 \Delta t$ and so on, using for every time step a full Newton-Raphson procedure. Finally, a finite difference scheme is used to define the temporal evolution during a finite time step and the time derivative:

$$
\begin{aligned}
x_{i} & =(1-\theta) x_{i}^{t}+\theta x_{i}^{t+\Delta t} \\
p & =(1-\theta) p^{t}+\theta p^{t+\Delta t} \\
\dot{p}^{t+\Delta t} & =\frac{p^{t+\Delta t}-p^{t}}{\Delta t}=\frac{\Delta p}{\Delta t}
\end{aligned}
$$

Knowing now the time evolution of all the variables, the field equations have to be met. A weighted residual method in time with point collocation [33] is used here. The choice of the $\theta$ value is not trivial. It is possible to show for linear system that the problem is unconditionally stable for $\theta \geqslant 0.5$ [33]. However, the system of equations considered here are highly non-linear and no theoretical proofs can assess that the previous conclusion is still valid in that case. The practice shows that extrapolation of the previous conclusions give generally good results. In the following, a complete implicit scheme is chosen $(\theta=1)$.

\section{LINEARIZATION OF THE FIELD EQUATIONS}

In order to obtain a full Newton-Raphson algorithm, the idea is to define a linear auxiliary problem deriving from the continuum one (instead of the discretized one as it is more usually done) similarly to the work of Borja [32]. This approach gives the same results than standard FEM procedure but make the linearization easier, especially for coupled problem in large strain 
formulation. Assuming known the configuration $\Omega^{t}$ at time $t$ in equilibrium with the boundary conditions, the objective is to find the new configuration at the end of the time step $(\tau=t+\Delta t)$. A guess of the solutions gives us $\Omega^{\tau 1}$ for which the equilibrium is not met $\left(\Omega^{\tau 1}\right.$ is assumed to be known). Some residuals can be defined according to the following equations:

$$
\begin{gathered}
\int_{\Omega^{\tau 1}}\left(\sigma_{i j}^{\tau 1} \frac{\partial u_{i}^{*}}{\partial x_{j}^{\tau 1}}+\Sigma_{i j k}^{\tau 1} \frac{\partial v_{i j}^{*}}{\partial x_{k}^{\tau 1}}\right) \mathrm{d} \Omega^{\tau 1}-\int_{\Omega^{\tau 1}} \lambda_{i j}^{\tau 1}\left(\frac{\partial u_{i}^{*}}{\partial x_{j}^{\tau 1}}-v_{i j}^{*}\right) \mathrm{d} \Omega^{\tau 1} \\
-\int_{\Omega^{\tau 1}}\left(\varrho^{S}\left(1-\phi^{\tau 1}\right)+\varrho^{w, \tau 1} \phi^{\tau 1}\right) g_{i} u_{i}^{*} \mathrm{~d} \Omega^{\tau 1}-\int_{\Gamma_{\sigma}^{\tau 1}}\left(\bar{t}_{i} u_{i}^{*}+\bar{T}_{i} v_{i k}^{*} n_{k}^{\tau 1}\right) \mathrm{d} \Gamma^{\tau 1}=R^{\tau 1} \\
\int_{\Omega^{\tau 1}} \lambda_{i j}^{*}\left(\frac{\partial u_{i}}{\partial x_{j}^{\tau 1}}-v_{i j}^{\tau 1}\right) \mathrm{d} \Omega^{\tau 1}=S^{\tau 1} \\
\int_{\Omega^{\tau 1}}\left(\dot{M}^{\tau 1} p^{\star}-m_{i}^{\tau 1} \frac{\partial p^{\star}}{\partial x_{i}^{\tau 1}}\right) \mathrm{d} \Omega^{\tau 1}-\int_{\Omega^{\tau 1}} Q^{\tau 1} p^{\star} \mathrm{d} \Omega^{\tau 1}+\int_{\Gamma_{q}^{\tau 1}} \bar{q}^{\tau 1} p^{\star} \mathrm{d} \Gamma^{\tau 1}=W^{\tau 1}
\end{gathered}
$$

The aim is now to find a new configuration $\Omega^{\tau 2}$, close to $\Omega^{\tau 1}$, for which the residuals $R^{\tau 2}, S^{\tau 2}$ and $W^{\tau 2}$ vanish $\left(\Omega^{\tau 2}\right.$ is the following guess of the configuration $\Omega$ at time $\tau$ ). In order to get the linear auxiliary problem, the field equations corresponding to $\Omega^{\tau 2}$ are rewritten in configuration $\Omega^{\tau 1}$ and the resulting equations are substracted from the corresponding equations (26)-(28). This yields:

$$
\begin{gathered}
\int_{\Omega^{\tau 1}} \frac{\partial u_{i}^{*}}{\partial x_{l}^{\tau 1}}\left(\sigma_{i j}^{\tau 2} \frac{\partial x_{l}^{\tau 1}}{\partial x_{j}^{\tau 2}} \operatorname{det} F-\sigma_{i l}^{\tau 1}\right)+\frac{\partial v_{i j}^{*}}{\partial x_{l}^{\tau 1}}\left(\Sigma_{i j k}^{\tau 2} \frac{\partial x_{l}^{\tau 1}}{\partial x_{k}^{\tau 2}} \operatorname{det} F-\Sigma_{i j l}^{\tau 1}\right) \mathrm{d} \Omega^{\tau 1} \\
-\int_{\Omega^{\tau 1}} \frac{\partial u_{i}^{*}}{\partial x_{l}^{\tau 1}}\left(\lambda_{i j}^{\tau 2} \frac{\partial x_{l}^{\tau 1}}{\partial x_{j}^{\tau 2}} \operatorname{det} F-\lambda_{i l}^{\tau 1}\right)+v_{i j}^{*}\left(\lambda_{i j}^{\tau 2} \operatorname{det} F-\lambda_{i l}^{\tau 1}\right) \mathrm{d} \Omega^{\tau 1} \\
-\int_{\Omega^{\tau 1}} u_{i}^{*}\left(\varrho^{\operatorname{mix}, \tau 2} \operatorname{det} F-\varrho^{\operatorname{mix}, \tau 1}\right) g_{i} \mathrm{~d} \Omega^{\tau 1}=-R^{\tau 1} \\
\int_{\Omega^{\tau 1}} \lambda_{i j}^{*}\left[\left(\frac{\partial u_{i}^{\tau 2}}{\partial x_{k}^{\tau 1}} \frac{\partial x_{k}^{\tau 1}}{\partial x_{j}^{\tau 2}} \operatorname{det} F-\frac{\partial u_{i}^{\tau 1}}{\partial x_{k}^{\tau 1}}\right)-\left(v_{i j}^{\tau 2} \operatorname{det} F-v_{i j}^{\tau 1}\right)\right] \mathrm{d} \Omega^{\tau 1}=-S^{\tau 1} \\
\int_{\Omega^{\tau 1}}\left(\dot{M}^{\tau 2} \operatorname{det} F-\dot{M}^{\tau 1}\right) p^{\star}-\frac{\partial p^{\star}}{\partial x_{l}^{\tau 1}}\left(m_{i}^{\tau 2} \frac{\partial x_{l}^{\tau 1}}{\partial x_{i}^{\tau 2}} \operatorname{det} F-m_{l}^{\tau 1}\right) \mathrm{d} \Omega^{\tau 1}=-W^{\tau 1}
\end{gathered}
$$

where we assume that $g_{i}, \bar{t}_{i}, \bar{q}$ and $Q$ are position independent and that double forces $\bar{T}_{i}$ vanish. $\operatorname{det} F$ is the Jacobian of $F_{i j}=\partial x_{i}^{\tau 2} / \partial x_{j}^{\tau 1}$. Let us define:

$$
\mathrm{d} u_{j}^{\tau 1}=x_{j}^{\tau 2}-x_{j}^{\tau 1}
$$




$$
\begin{aligned}
\mathrm{d} v_{i j}^{\tau 1} & =v_{i j}^{\tau 2}-v_{i j}^{\tau 1} \\
\mathrm{~d} \lambda_{i l}^{\tau 1} & =\lambda_{i l}^{\tau 2}-\lambda_{i l}^{\tau 1} \\
\mathrm{~d} p^{\tau 1} & =p^{\tau 2}-p^{\tau 1} \\
\mathrm{~d} \sigma_{i l}^{\tau 1} & =\sigma_{i l}^{\tau 2}-\sigma_{i l}^{\tau 1} \\
\mathrm{~d} \Sigma_{i k l}^{\tau 1} & =\Sigma_{i k l}^{\tau 2}-\Sigma_{i k l}^{\tau 1} \\
\mathrm{~d} \phi^{\tau 1} & =\phi^{\tau 2}-\phi^{\tau 1} \\
\mathrm{~d} \rho^{w, \tau 1} & =\rho^{w, \tau 2}-\rho^{w, \tau 1}
\end{aligned}
$$

Using a Taylor expansion of Equations (29)-(31) and discarding terms of degree greater than one yields after some algebra (see Reference [36] for more details) to the following linearized equations, which allow us to find the corrections of the displacement fields $\mathrm{d} u_{i}, \mathrm{~d} v_{i j}, \mathrm{~d} \lambda_{i j}$ and the corrections of the pressure $\mathrm{d} p$ to be added to their respective current values to obtain a new current configuration, and a new pore pressure field closer to a well-balanced configuration:

$$
\begin{aligned}
& \int_{\Omega^{\tau 1}} \frac{\partial u_{i}^{\star}}{\partial x_{l}^{\tau 1}}\left(\mathrm{~d} \sigma_{i l}^{\tau 1}-\sigma_{i j}^{\tau 1} \frac{\partial \mathrm{d} u_{l}}{\partial x_{j}^{\tau 1}}+\sigma_{i l}^{\tau 1} \frac{\partial \mathrm{d} u_{m}}{\partial x_{m}^{\tau 1}}\right)+\frac{\partial v_{i k}^{\star}}{\partial x_{l}^{\tau 1}}\left(\mathrm{~d} \Sigma_{i k l}^{\tau 1}-\Sigma_{i k j}^{\tau 1} \frac{\partial \mathrm{d} u_{l}}{\partial x_{j}^{\tau 1}}+\Sigma_{i k l}^{\tau 1} \frac{\partial \mathrm{d} u_{m}}{\partial x_{m}^{\tau 1}}\right) \mathrm{d} \Omega^{\tau 1} \\
& -\int_{\Omega^{\tau 1}} \frac{\partial u_{i}^{\star}}{\partial x_{l}^{\tau 1}}\left(\mathrm{~d} \lambda_{i l}^{\tau 1}-\lambda_{i j}^{\tau 1} \frac{\partial \mathrm{d} u_{l}}{\partial x_{j}^{\tau 1}}+\lambda_{i l}^{\tau 1} \frac{\partial \mathrm{d} u_{m}}{\partial x_{m}^{\tau 1}}\right)-v_{i j}^{\star}\left(\mathrm{d} \lambda_{i l}^{\tau 1}+\lambda_{i l}^{\tau 1} \frac{\partial \mathrm{d} u_{m}}{\partial x_{m}^{\tau 1}}\right) \mathrm{d} \Omega^{\tau 1} \\
& -\int_{\Omega^{\tau 1}} u_{i}^{\star}\left[\varrho^{\operatorname{mix}, \tau 1} \frac{\partial \mathrm{d} u_{m}}{\partial x_{m}^{\tau 1}}-\left(\varrho^{s, \tau 1}-\varrho^{w, \tau 1}\right) \mathrm{d} \phi^{\tau 1}+\mathrm{d} \varrho^{w, \tau 1} \phi^{\tau 1}\right] g_{i} \mathrm{~d} \Omega^{\tau 1}=-R^{\tau 1} \\
& \int_{\Omega^{\tau 1}} \lambda_{i j}^{*}\left[\left(\frac{\partial u_{i}^{\tau 1}}{\partial x_{j}^{\tau 1}}-v_{i j}^{\tau 1}\right) \frac{\partial \mathrm{d} u_{m}^{\tau 1}}{\partial x_{m}^{\tau 1}}+\frac{\partial \mathrm{d} u_{i}^{\tau 1}}{\partial x_{j}^{\tau 1}}-\frac{\partial u_{i}^{\tau 1}}{\partial x_{k}^{\tau 1}} \frac{\partial \mathrm{d} u_{k}^{\tau 1}}{\partial x_{j}^{\tau 1}}-\mathrm{d} v_{i j}^{\tau 1}\right] \mathrm{d} \Omega^{\tau 1}=-S^{\tau 1} \\
& \int_{\Omega^{\tau 1}} p^{\star}\left(\mathrm{d} \varrho^{w, \tau 1} \frac{\phi^{\tau 1}}{k^{w}} \dot{p}^{\tau 1}+\varrho^{w, \tau 1} \frac{\mathrm{d} \phi^{\tau 1}}{k^{w}} \dot{p}^{\tau 1}+\varrho^{w, \tau 1} \frac{\phi^{\tau 1}}{k^{w}} \frac{\mathrm{d} p^{\tau 1}}{\Delta t}\right. \\
& \left.+\mathrm{d} \varrho^{w, \tau 1} \frac{\dot{\Omega}^{\tau 1}}{\Omega^{\tau 1}}+\varrho^{w, \tau 1}\left(\frac{\partial \mathrm{d} u_{m}^{\tau 1}}{\partial x_{m}^{\tau 1}} \frac{1}{\Delta t}-\frac{\dot{\Omega}^{\tau 1}}{\Omega^{\tau 1}} \frac{\partial \mathrm{d} u_{m}^{\tau 1}}{\partial x_{m}^{\tau 1}}\right)+\dot{M}^{\tau 1} \frac{\partial \mathrm{d} u_{m}^{\tau 1}}{\partial x_{m}^{\tau 1}}\right) \mathrm{d} \Omega^{\tau 1} \\
& \quad-\int_{\Omega^{\tau 1}} \frac{\partial p^{\star}}{\partial x_{l}^{\tau 1}}\left(-\mathrm{d} \varrho^{w, \tau 1} \frac{\kappa}{\mu}\left(\frac{\partial p^{\tau 1}}{\partial x_{l}^{\tau 1}}+\varrho^{w, \tau 1} g_{l}\right)-\varrho^{w, \tau 1} \frac{\kappa}{\mu}\left(\frac{\partial \mathrm{d} p^{\tau 1}}{\partial x_{l}^{\tau 1}}+\mathrm{d} \varrho^{w, \tau 1} g_{l}\right)\right. \\
& \left.+\varrho^{w, \tau 1} \frac{\kappa}{\mu} \frac{\partial \mathrm{d} u_{j}^{\tau 1}}{\partial x_{l}^{\tau 1}} \frac{\partial p^{\tau 1}}{\partial x_{j}^{\tau 1}}-m_{j}^{\tau 1} \frac{\partial \mathrm{d} u_{l}^{\tau 1}}{\partial x_{j}^{\tau 1}}+m_{l}^{\tau 1} \frac{\partial \mathrm{d} u_{m}^{\tau 1}}{\partial x_{m}^{\tau 1}}\right) \mathrm{d} \Omega^{\tau 1}=-W^{\tau 1}
\end{aligned}
$$


If we assume the following relationship coming from a linearization of Equations (6), (8) and (3) (for the last three ones), the preceding system can be expressed as a function of the unknowns $\mathrm{d} u_{i}, \mathrm{~d} v_{i j}, \mathrm{~d} \lambda_{i j}$ and $\mathrm{d} p$

$$
\begin{aligned}
\mathrm{d} \Sigma_{i k l}^{\tau 1} & =D_{i k l j m n}^{\tau 1} \frac{\partial \mathrm{d} v_{j m}^{\tau 1}}{\partial x_{n}^{\tau 1}} \\
\mathrm{~d} \sigma_{i l}^{\tau 1} & =\mathrm{d} \sigma_{i l}^{\prime \tau 1}-\mathrm{d} p^{\tau 1} \delta_{i l}=C_{i l k j}^{\tau 1} \frac{\partial \mathrm{d} u_{k}^{\tau 1}}{\partial x_{j}^{\tau 1}}-\mathrm{d} p^{\tau 1} \delta_{i l} \\
\mathrm{~d} \varrho^{w, \tau 1} & =\frac{\varrho^{w, \tau 1}}{k^{w}} \mathrm{~d} p^{\tau 1} \\
\mathrm{~d} \phi^{\tau 1} & =\left(1-\phi^{\tau 1}\right) \frac{\mathrm{d} \Omega^{\tau 1}}{\Omega^{\tau 1}}=\left(1-\phi^{\tau 1}\right) \frac{\partial \mathrm{d} u_{m}^{\tau 1}}{\partial x_{m}^{\tau 1}}
\end{aligned}
$$

It should be pointed out that matrices $C_{i l k j}^{\tau 1}$ and $D_{i k l j m n}^{\tau 1}$ are computed through a consistent linearization of the constitutive law integration algorithm. Consistent linearization operators are now well known for first gradient law [38] and they are directly adapted to the second gradient constitutive model like in Reference [35]. Equations (40)-(42) have different contributions coming from geometrical non-linear effects (since we distinguished $\Omega^{\tau 1}$ and $\Omega^{\tau 2}$ in all the developments), from the microstructure terms and finally from the couplings between pore pressure and the mechanical problem. These contributions will be highlighted in the next section, where the formulation of a bi-dimensional finite element is detailed.

\section{COUPLED FINITE ELEMENT FORMULATION}

The field equations (40)-(42) are spatially discretized using 2D plane strain isoparametric finite elements with eight nodes for $u_{i}$ and $p$, four nodes for $v_{i j}$ and one node for $\lambda_{i j}$ (Figure 1). The usual quadratic serendipity shape function [33] are used for $u_{i}$ and $p$. The linear shape functions are used for $v_{i j}$, and $\lambda_{i j}$ is assumed to be constant. The choice of quadratic shape function for the pressure field comes from the experience of the authors in classical poromechanic problems,

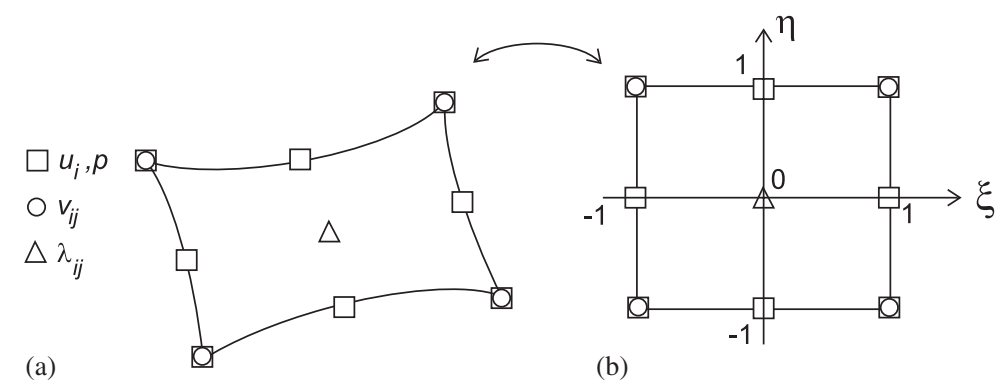

Figure 1. Quadrilateral element and parent element: (a) quadrilateral element; and (b) parent element. 
for which this kind of function has been used without any difficulties. A corresponding FE with linear shape functions for the pressure will be experienced in the future in order to compare its results with the one obtained with the element used in this paper.

In order to define the local element stiffness matrix, Equations (40)-(42) are rewritten in a matricial form

$$
\int_{\Omega^{\tau 1}}\left[U_{(x, y)}^{*, \tau 1}\right]^{\mathrm{T}}\left[E^{\tau 1}\right]\left[\mathrm{d} U_{(x, y)}^{\tau 1}\right] \mathrm{d} \Omega^{\tau 1}=-R^{\tau 1}-S^{\tau 1}-W^{\tau 1}
$$

where $\left[\mathrm{d} U_{(x, y)}^{\tau 1}\right]$ is defined in Equation (48) and $\left[U_{(x, y)}^{*, \tau 1}\right]$ has the same structure with corresponding virtual quantities:

$$
\begin{aligned}
{\left[\mathrm{d} U_{(x, y)}^{\tau 1}\right] \equiv } & {\left[\frac{\partial \mathrm{d} u_{1}^{\tau 1}}{\partial x_{1}^{\tau 1}} \frac{\partial \mathrm{d} u_{1}^{\tau 1}}{\partial x_{2}^{\tau 1}} \frac{\partial \mathrm{d} u_{2}^{\tau 1}}{\partial x_{1}^{\tau 1}} \frac{\partial \mathrm{d} u_{2}^{\tau 1}}{\partial x_{2}^{\tau 1}} \mathrm{~d} u_{1}^{\tau 1} \mathrm{~d} u_{2}^{\tau 1} \frac{\partial \mathrm{d} p^{\tau 1}}{\partial x_{1}^{\tau 1}} \frac{\partial \mathrm{d} p^{\tau 1}}{\partial x_{2}^{\tau 1}} \mathrm{~d} p^{\tau 1}\right.} \\
& \left.\frac{\partial \mathrm{d} v_{11}^{\tau 1}}{\partial x_{1}^{\tau 1}} \frac{\partial \mathrm{d} v_{11}^{\tau 1}}{\partial x_{2}^{\tau 1}} \frac{\partial \mathrm{d} v_{12}^{\tau 1}}{\partial x_{1}^{\tau 1}} \cdots \frac{\partial \mathrm{d} v_{22}^{\tau 1}}{\partial x_{2}^{\tau 1}} \mathrm{~d} v_{11}^{\tau 1} \mathrm{~d} v_{12}^{\tau 1} \mathrm{~d} v_{21}^{\tau 1} \mathrm{~d} v_{22}^{\tau 1} \mathrm{~d} \lambda_{11}^{\tau 1} \mathrm{~d} \lambda_{12}^{\tau 1} \mathrm{~d} \lambda_{21}^{\tau 1} \mathrm{~d} \lambda_{22}^{\tau 1}\right]
\end{aligned}
$$

The finite element spatial discretization is introduced in Equation (47) using the transformation matrices $\left[T^{\tau 1}\right]$ and $[B]$, which connect $\left[\mathrm{d} U_{(x, y)}^{\tau 1}\right]$ to the nodal variables $\left[\mathrm{d} U_{\text {Node }}^{\tau 1}\right]$ :

$$
\left[\mathrm{d} U_{(x, y)}^{\tau 1}\right]=\left[T^{\tau 1}\right]\left[\mathrm{d} U_{(\xi, \eta)}^{\tau 1}\right]
$$

and

$$
\left[\mathrm{d} U_{(\xi, \eta)}^{\tau 1}\right]=[B]\left[\mathrm{d} U_{\text {Node }}^{\tau 1}\right]
$$

Integration of Equation (47) on one finite element yields

$$
\begin{aligned}
& {\left[U_{\text {node }}^{*}\right]^{\mathrm{T}} \int_{-1}^{1} \int_{-1}^{1}[B]^{\mathrm{T}}\left[T^{\tau 1}\right]^{\mathrm{T}}\left[E^{\tau 1}\right]\left[T^{\tau 1}\right][B] \operatorname{det} J^{\tau 1} \mathrm{~d} \xi \mathrm{d} \eta\left[\mathrm{d} U_{\text {node }}^{t}\right]} \\
& \equiv\left[U_{\text {node }}^{*}\right]^{\mathrm{T}}\left[k^{\tau 1}\right]\left[\mathrm{d} U_{\text {node }}^{\tau 1}\right]
\end{aligned}
$$

where $\left[k^{\tau 1}\right]$ is the local element stiffness matrix, $J^{\tau 1}$ is the Jacobian matrix of the mapping from $(\xi, \eta)$ to $(x, y)$ and $\left[\mathrm{d} U_{\text {node }}^{\tau 1}\right]$ has the following definition:

$$
\begin{aligned}
{\left[\mathrm{d}_{\text {Node }}^{\tau 1}\right]^{\mathrm{T}}=} & {\left[\mathrm{d} u_{1(-1,-1)}^{\tau 1} \mathrm{~d} u_{2(-1,-1)}^{\tau 1} \mathrm{~d} p_{(-1,-1)}^{\tau 1} \mathrm{~d} v_{11(-1,-1)}^{\tau 1} \mathrm{~d} v_{12(-1,-1)}^{\tau 1} \mathrm{~d} v_{21(-1,-1)}^{\tau 1}\right.} \\
& \times \mathrm{d} v_{22(-1,-1)}^{\tau 1} \mathrm{~d} u_{1(-1,0)}^{\tau 1} \mathrm{~d} u_{2(-1,0)}^{\tau 1} \mathrm{~d} p_{(-1,0)}^{\tau 1} \mathrm{~d} u_{1(-1,1)}^{\tau 1} \mathrm{~d} u_{2(-1,1)}^{\tau 1} \mathrm{~d} p_{(-1,1)}^{\tau 1} \\
& \times \mathrm{d} v_{11(-1,1)}^{\tau 1} \mathrm{~d} v_{12(-1,1)}^{\tau 1} \mathrm{~d} v_{21(-1,1)}^{\tau 1} \mathrm{~d} v_{22(-1,1)}^{\tau 1} \mathrm{~d} u_{1(0,-1)}^{\tau 1} \mathrm{~d} u_{2(0,-1)}^{\tau 1} \mathrm{~d} p_{(0,-1)}^{\tau 1} \\
& \times \mathrm{d} \lambda_{11(0,0)}^{\tau 1} \mathrm{~d} \lambda_{12(0,0)}^{\tau 1} \mathrm{~d} \lambda_{21(0,0)}^{\tau 1} \mathrm{~d} \lambda_{22(0,0)}^{\tau 1} \mathrm{~d} u_{1(0,1)}^{\tau 1} \mathrm{~d} u_{2(0,1)}^{\tau 1} \mathrm{~d} p_{(0,1)}^{\tau 1} \\
& \times \mathrm{d} u_{1(1,-1)}^{\tau 1} \mathrm{~d} u_{2(1,-1)}^{\tau 1} \mathrm{~d} p_{(1,-1)}^{\tau 1} \mathrm{~d} v_{11(1,-1)}^{\tau 1} \mathrm{~d} v_{12(1,-1)}^{\tau 1} \mathrm{~d} v_{21(1,-1)}^{\tau 1} \mathrm{~d} v_{22(1,-1)}^{\tau 1}
\end{aligned}
$$




$$
\begin{aligned}
& \times \mathrm{d} u_{1(1,0)}^{\tau 1} \mathrm{~d} u_{2(1,0)}^{\tau 1} \mathrm{~d} p_{(1,0)}^{\tau 1} \mathrm{~d} u_{1(1,1)}^{\tau 1} \mathrm{~d} u_{2(1,1)}^{\tau 1} \mathrm{~d} p_{(1,1)}^{\tau 1} \\
& \left.\times \mathrm{d} v_{11(1,1)}^{\tau 1} \mathrm{~d} v_{12(1,1)}^{\tau 1} \mathrm{~d} v_{21(1,1)}^{\tau 1} \mathrm{~d} v_{22(1,1)}^{\tau 1}\right]
\end{aligned}
$$

$\left[E^{\tau 1}\right]$ is a $25 \times 25$ matrix that contains all the terms of Equations $(40)-(42)$ :

$$
\left[E^{\tau 1}\right]=\left[\begin{array}{cccccc}
E 1_{(4 \times 4)}^{\tau 1} & 0_{(4 \times 2)} & K_{W M_{(4 \times 3)}}^{\tau 1} & 0_{(4 \times 8)} & 0_{(4 \times 4)} & -I_{(4 \times 4)} \\
G 1_{(2 \times 4)}^{\tau 1} & 0_{(2 \times 2)} & G 2_{(2 \times 3)}^{\tau 1} & 0_{(2 \times 8)} & 0_{(2 \times 4)} & 0_{(2 \times 4)} \\
K_{M W_{(3 \times 4)} 1_{(3 \times 2)}} & 0_{W W_{(3 \times 3)}}\left(0_{(3 \times 8)}\right. & 0_{(3 \times 4)} & 0_{(3 \times 4)} \\
E 2_{(8 \times 4)}^{\tau 1} & 0_{(8 \times 2)} & 0_{(8 \times 3)} & D_{(8 \times 8)}^{\tau 1} & 0_{(8 \times 4)} & 0_{(8 \times 4)} \\
E 3_{(4 \times 4)}^{\tau 1} & 0_{(4 \times 2)} & 0_{(4 \times 3)} & 0_{(4 \times 8)} & 0_{(4 \times 4)} & I_{(4 \times 4)} \\
E 4_{(4 \times 4)}^{\tau 1} & 0_{(4 \times 2)} & 0_{(4 \times 3)} & 0_{(4 \times 8)} & -I_{(4 \times 4)} & 0_{(4 \times 4)}
\end{array}\right]
$$

Matrices $E 1, E 2, E 3, E 4$ and $D$ are identical to these corresponding to local second gradient for monophasic medium (see Reference [36] for details). Matrix $K_{W W}$ is the classical stiffness matrix for a flow problem. $K_{W M}, K_{M W}$ contain all the couplings terms appearing between the mechanical problem and the flow one. Matrices $G 1$ and $G 2$ are related to the contribution of gravity volume force. In all these matrices detailed hereafter, all terms coming from the large strain formulation are written between braces.

$$
\left[K_{W W(3 \times 3)}^{\tau 1}\right]=\left[\begin{array}{ccc}
\varrho^{w, \tau 1} \frac{\kappa}{\mu} & 0 & \frac{\varrho^{w, \tau 1}}{k_{w}} \frac{\kappa}{\mu}\left(\frac{\partial p^{\tau 1}}{\partial x_{1}^{\tau 1}}+\varrho^{w, \tau 1} g_{1}\right)+\varrho^{w, \tau 1} \frac{\kappa}{\mu} \frac{\varrho^{w, \tau 1}}{k_{w}} g_{1} \\
0 & \varrho^{w, \tau 1} \frac{\kappa}{\mu} & \frac{\varrho^{w, \tau 1}}{k_{w}} \frac{\kappa}{\mu}\left(\frac{\partial p^{\tau 1}}{\partial x_{2}^{\tau 1}}+\varrho^{w, \tau 1} g_{2}\right)+\varrho^{w, \tau 1} \frac{\kappa}{\mu} \frac{\varrho^{w, \tau 1}}{k_{w}} g_{2} \\
0 & 0 & \frac{\varrho^{w, \tau 1}}{k_{w}} \frac{\phi^{\tau 1}}{k^{w}} \dot{p}^{\tau 1}+\varrho^{w, \tau 1} \frac{\phi^{\tau 1}}{k^{w}} \frac{1}{\Delta t}+\frac{\varrho^{w, \tau 1}}{k_{w}} \frac{\dot{\Omega}^{\tau 1}}{\Omega^{\tau 1}}
\end{array}\right]
$$




$$
\begin{aligned}
& {\left[K_{W M(4 \times 3)}^{\tau 1}\right]=\left[\begin{array}{ccc}
0 & 0 & -1 \\
0 & 0 & 0 \\
0 & 0 & 0 \\
0 & 0 & -1
\end{array}\right]} \\
& {\left[G 1_{(2 \times 4)}^{\tau 1}\right]=\left[\begin{array}{llll}
B & 0 & 0 & B \\
B & 0 & 0 & B
\end{array}\right]} \\
& {\left[G 2_{(2 \times 3)}^{\tau 1}\right]=\left[\begin{array}{ccc}
0 & 0 & -\varrho^{w, \tau 1} \frac{\phi^{\tau 1}}{k^{w}} g_{1} \\
0 & 0 & -\varrho^{w, \tau 1} \frac{\phi^{\tau 1}}{k^{w}} g_{2}
\end{array}\right]}
\end{aligned}
$$

where

$$
A=\varrho^{w, \tau 1}\left(\frac{1-\phi^{\tau 1}}{k^{w}} \dot{p}^{\tau 1}+\frac{1}{\Delta t}-\left\{\frac{\dot{\Omega}^{\tau 1}}{\Omega^{\tau 1}}\right\}\right)
$$

and

$$
B=\left\{-\varrho^{\operatorname{mix}, \tau 1}\right\}+\left(\varrho^{s, \tau 1}-\varrho^{w, \tau 1}\right)\left(1-\phi^{\tau 1}\right)
$$

In Equation (47), the residual terms $R^{\tau 1}, S^{\tau 1}$ and $W^{\tau 1}$ are computed for each element thanks to the following relationship:

$$
\begin{gathered}
-R^{\tau 1}-S^{\tau 1}-W^{\tau 1}=P_{e}^{t^{*}}-\left[U_{\text {node }}^{*}\right]^{\mathrm{T}} \int_{-1}^{1} \int_{-1}^{1}[B]^{\mathrm{T}}\left[T^{t}\right]^{\mathrm{T}}\left[\sigma^{t}\right] . \operatorname{det} J^{t} \mathrm{~d} \xi \mathrm{d} \eta \\
\equiv\left[U_{\text {node }}^{*}\right]\left[f_{H E}^{t}\right] \\
{\left[\sigma^{\tau 1}\right]=\left[\sigma_{11}^{\tau 1}-\lambda_{11}^{\tau 1} \cdots \sigma_{22}^{\tau 1}-\lambda_{22}^{\tau 1}-\varrho^{\mathrm{mix}, \tau 1} g_{1}-\varrho^{\mathrm{mix}, \tau 1} g_{2}-m_{1}^{\tau 1}-m_{2}^{\tau 1} \dot{M}^{\tau 1}\right.} \\
\left.\times \Sigma_{111}^{\tau 1} \cdots \Sigma_{222}^{\tau 1} \lambda_{11}^{\tau 1} \cdots \lambda_{22}^{\tau 1} \frac{\partial u_{1}^{\tau 1}}{\partial x_{1}^{\tau 1}}-v_{11}^{\tau 1} \cdots \frac{\partial u_{2}^{\tau 1}}{\partial x_{2}^{\tau 1}}-v_{22}^{\tau 1}\right]
\end{gathered}
$$

where $\left[f_{H E}^{t}\right]$ are the elementary out of balance forces. The external virtual power $P_{e}^{t^{*}}$ has two contributions: the body forces and the boundary forces (for the mechanical and flow problems), except the term related to gravity volume force, which is introduced in the $\left[\sigma^{\tau 1}\right]$ vector.

The global stiffness matrix and out of balance force can be obtained by assembling the elementary matrices given by Equations (51) and (61). After solving the resulting auxiliary linear system, a new configuration is found and the equilibrium is checked. Different approaches exist to solve coupled equations problems. The staggered procedure concept consists in solving 
Table I. The finite step algorithm for one loading step.

1. Initial configuration:

stress $\sigma^{t-\Delta t}$, double stress $\Sigma^{t-\Delta t}$, co-ordinates $x^{t-\Delta t}$, pore pressure $p^{t-\Delta t}$

2. Assumption on the final configuration and the final pressure

Update nodal co-ordinates: $x^{t_{n}}$, for $n:=1$

3. Beginning of the iteration $n$ :

4. For each element

- For each integration point:

- compute the strain rate, the rotation rate, the second gradient rate and the pore pressure rate,

$\circ$ compute $\Delta \sigma_{n}^{\prime}, \Delta \Sigma_{n}$ and $m_{n}$ using constitutive equations and the flow model,

$\circ$ update the effective stress and the double stress

$\sigma^{\prime t_{n}}:=\sigma^{\prime t-\Delta t}+\Delta \sigma_{n}^{\prime}$,

$\Sigma^{t_{n}}:=\Sigma^{t-\Delta t}+\Delta \Sigma_{n}$,

○ compute the total stress $\sigma^{t_{n}}:=\sigma^{\prime t_{n}}-p^{t_{n}}$,

- compute the compliance matrices by mean of perturbation methods $\left[C_{(4 \times 4)}^{t_{n}}\right]$ and $\left[D_{(8 \times 8)}^{t_{n}}\right]$

- Compute the element stiffness matrix $\left[K^{t_{n}}\right]$

- Compute the element out of balance forces $\left[f_{H E}^{t_{n}}\right]$

5. Compute the global stiffness matrix $\left[K^{t_{n}}\right]$

6. Compute the global out of balance forces $\left[F_{H E}^{t_{n}}\right]$

7. Compute $\left[\delta U_{\text {node }}^{t_{n}}\right]$ by solving $\left[K^{t_{n}}\right]\left[\delta U_{\text {node }}^{t_{n}}\right]=-\left[F_{H E}^{t_{n}}\right]$

8. Check the accuracy of the computed solution

- If convergence: go to 9

- If no convergence: update the new assumed final configuration and the final pore pressure, $n:=n+1$, go to 3

9. End of the step

a first block of equations related to a variable field while the other are kept constant. In a second step, the second block of equations is solved while the updated variable fields remain fixed (see References $[5,39,40]$ ). Here a monolithic procedure is chosen where the full stiffness matrix is computed at each iteration of the Newton-Raphson procedure.

Table I shows the algorithm used to solve one loading step of a local second gradient coupled application.

\section{NUMERICAL APPLICATION OF LOCAL SECOND GRADIENT COUPLED MODEL}

Biaxial experiments are known to exhibit clearly strain localization either in soil or rock samples (see for instance Reference [9]). These tests have been performed and shear banding has been observed in drained or undrained conditions, in both contractive and dilative specimens [10].

Modelling such a plane strain compression test is proposed here in order to study the regularization properties of the local second gradient model for a biphasic medium. In this first approach, a very simple constitutive equation has been chosen for the skeleton. A classical model 
based on the Prandtl-Reuss elasto-plastic model gives the effective stress tensor $\sigma_{i j}^{\prime}$ as follows:

$$
\begin{aligned}
& \dot{\sigma}_{m}^{\prime}=3 K \dot{e} \\
& \tilde{\hat{\sigma}}_{i j}^{\prime}= \begin{cases}2 G_{1} \dot{\hat{\varepsilon}}_{i j} & \left(\|\hat{\varepsilon}\|<e_{\lim }\right) \\
2 G_{1}\left(\dot{\hat{\varepsilon}}_{i j}-\frac{G_{1}-G_{2}}{G_{1}} \frac{\hat{\sigma}_{k l}^{\prime}}{\left\|\hat{\sigma}^{\prime}\right\|^{2}} \hat{\hat{\sigma}}_{i j}^{\prime}\right) & \left(\|\hat{\varepsilon}\|>e_{\lim }\right)\end{cases}
\end{aligned}
$$

$K$ is the bulk modulus, $G_{1}$ and $G_{2}$ are the shear moduli before and after the peak, respectively, $\quad \dot{\sigma}_{m}^{\prime}$ is the effective mean stress rate, $\dot{e}$ is the mean strain rate, $\dot{\hat{\varepsilon}}_{i j}$ is the deviatoric strain rate tensor and $\tilde{\hat{\sigma}}_{i j}^{\prime}$ is the Jaumann rate of the deviatoric effective Cauchy stress tensor. $\|\hat{\varepsilon}\|$ and $\left\|\hat{\sigma}^{\prime}\right\|$ are the second invariant of the deviatoric strain and effective stress, respectively, and $e_{\text {lim }}$ is a model parameter.

An exponential function is assumed for the evolution of shear modulus $G_{2}$ after the yield point, so that the material could reach its residual state smoothly:

$$
G_{2}=\bar{G}_{2} \exp \left(\frac{\bar{G}_{2}}{G_{1} e_{\text {lim }}-\hat{\sigma}_{\text {res }}^{\prime}}\left(\|\hat{\varepsilon}\|-e_{\text {lim }}\right)\right)
$$

$\bar{G}_{2}$ is the value of the shear modulus just after the peak and $\hat{\sigma}_{\text {res }}^{\prime}$ is the residual value of the second invariant of deviatoric effective stress tensor.

For the second gradient part of the model, a simple isotropic linear relationship deduced from Reference [22] is used. It depends only on one elastic parameter denoted $D$ :

$$
\left[\begin{array}{l}
\tilde{\Sigma}_{111} \\
\tilde{\Sigma}_{112} \\
\tilde{\Sigma}_{121} \\
\tilde{\Sigma}_{122} \\
\tilde{\Sigma}_{211} \\
\tilde{\Sigma}_{212} \\
\tilde{\Sigma}_{221} \\
\tilde{\Sigma}_{222}
\end{array}\right]=\left[\begin{array}{cccccccc}
D & 0 & 0 & 0 & 0 & \frac{D}{2} & \frac{D}{2} & 0 \\
0 & \frac{D}{2} & \frac{D}{2} & 0 & -\frac{D}{2} & 0 & 0 & \frac{D}{2} \\
0 & \frac{D}{2} & \frac{D}{2} & 0 & -\frac{D}{2} & 0 & 0 & \frac{D}{2} \\
0 & 0 & 0 & D & 0 & -\frac{D}{2} & -\frac{D}{2} & 0 \\
0 & -\frac{D}{2} & -\frac{D}{2} & 0 & D & 0 & 0 & 0 \\
\frac{D}{2} & 0 & 0 & -\frac{D}{2} & 0 & \frac{D}{2} & \frac{D}{2} & 0 \\
\frac{D}{2} & 0 & 0 & -\frac{D}{2} & 0 & \frac{D}{2} & \frac{D}{2} & 0 \\
0 & \frac{D}{2} & \frac{D}{2} & 0 & 0 & 0 & 0 & 0
\end{array}\right]\left[\begin{array}{c}
\frac{\partial \dot{v}_{11}}{\partial x_{1}} \\
\frac{\partial \dot{v}_{11}}{\partial x_{2}} \\
\frac{\partial \dot{v}_{12}}{\partial x_{1}} \\
\frac{\partial \dot{v}_{12}}{\partial x_{2}} \\
\frac{\partial \dot{v}_{21}}{\partial x_{1}} \\
\frac{\partial \dot{v}_{21}}{\partial x_{2}} \\
\frac{\partial \dot{v}_{22}}{\partial x_{1}} \\
\frac{\partial \dot{v}_{22}}{\partial x_{2}}
\end{array}\right]
$$




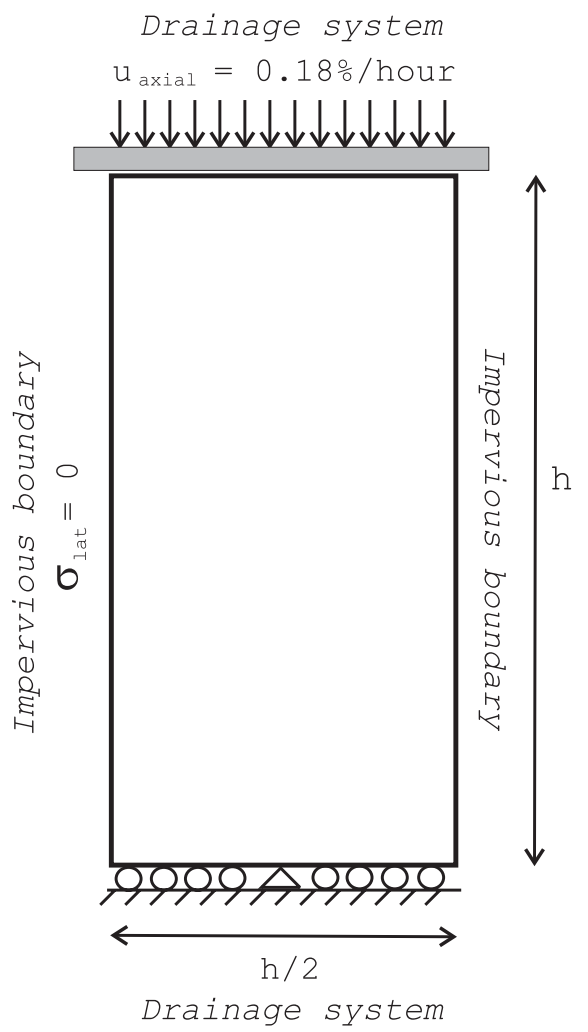

Figure 2. Sketch of the boundary value problem.

$\dot{v}_{i j}$ is the material time derivative of $v_{i j}, \tilde{\Sigma}_{i j k}$ is the Jaumann double stress rate defined by the following equation:

$$
\tilde{\Sigma}_{i j k}=\dot{\Sigma}_{i j k}+\Sigma_{l j k} \omega_{l i}+\Sigma_{i m k} \omega_{m j}+\Sigma_{i j p} \omega_{p k}
$$

where $\omega_{l i}$ is the spin tensor.

A drained strain-controlled bi-axial test is considered. Drained means here globally drained test. This means that the boundary drainage systems are open: fluid is free to flow out of or into the sample but overpressures can appear in the sample depending on the ratio between loading rate and permeability.

The loading strain rate is $0.18 \%$ per hour. The bottom plate is smooth, rigid and remains horizontal. The central point of this plate is horizontally fixed in order to avoid rigid body displacement. The top plate is also smooth and rigid, and a prescribed vertical displacement is then applied to every corresponding node of the mesh. No lateral confining pressure is prescribed. The external additional double forces per unit area $T_{i}$ are assumed to be equal to zero all along the boundaries. Pore pressures are fixed to their initial value at the top and bottom edges, in order to model the upper and lower drainage system. The pore fluid pressure is initially equal to the atmospheric pressure (Figure 2). 
Table II. Parameters of the models.

\begin{tabular}{lccccc}
\hline First & gradient model & Second gradient model & \multicolumn{2}{c}{ Flow model } \\
\hline$K$ & $97.3856 \mathrm{MPa}$ & $D$ & $500 \mathrm{~N}$ & \multirow{2}{*}{$\kappa$} & $10^{-19} / 10^{-12} \mathrm{~m}^{2}$ \\
$G_{1}$ & $50 \mathrm{MPa}$ & & $\rho^{w}$ & $1000 \mathrm{~kg} / \mathrm{m}^{3}$ \\
$\bar{G}_{2}$ & $-2 \mathrm{MPa}$ & & \multicolumn{2}{c}{$\phi$} & 0.15 \\
$e_{l}$ & 0.01 & & $k_{w}$ & $510^{-10} \mathrm{~Pa}^{-1}$ \\
$\hat{\sigma}_{\text {res }}$ & $0.2 \sigma_{\text {peak }} \mathrm{MPa}$ & & & $\mu_{w}$ & $0.001 \mathrm{~Pa} \mathrm{~s}$ \\
\hline
\end{tabular}

The parameters used for the simulations are listed in Table II. Two values of intrinsic permeability have been used and then allow to see the influence of that property on the localization pattern.

In order to exhibit strain localization, different procedures are available. The more general is the introduction of an imperfection. This could be a geometrical default [41], a material imperfection [35] or a small disturbing force [42]. In a different way, Chambon and co-workers [43] proposed an algorithm to search localized solutions in perfect sample using a random initialization of the strain rate at the beginning of the iterative procedure. In the following computations, a material imperfection is introduced in the bottom left finite element. In this element, the parameter $e_{\text {lim }}$ is $10 \%$ lower than the one used elsewhere.

For the first simulation, the intrinsic permeability is equal to $10^{-12} \mathrm{~m}^{2}$. For such values of loading rate and permeability, overpressures in the sample are very low and the corresponding loading curve is equivalent to the curve obtained with a monophasic local second gradient model (Figure 4). Figure 3 shows us the localization pattern clearly initiated by the material default and the water flows related to the drainage of the sample. The pore fluid flow seems to be independent of the developed shear band because the constitutive model introduces no dilatancy in the plastic regime and the permeability is very high in comparison with the strain rate.

Other simulations of biaxial experiment have been performed with a permeability value of $10^{-19} \mathrm{~m}^{2}$. In fact, for such a value, the loading rate is so fast that the drainage is not possible and the curve corresponds to an undrained biaxial test. Different mesh sizes have been used and Figure 4 shows us that the corresponding load-displacement curves are merged. Equivalent strains are plotted on Figure 6 for $20 \times 10,30 \times 15,40 \times 20$ elements meshes. The zone where strains are localized is clearly seen. Before the localization process, the strain in the sample is homogeneous. Just after localization, the geomaterial inside the shear band loads plastically, but it unloads elastically outside. This is quite clear in Figure 7: a square is plotted for each plastic Gauss point. This allows us to accurately measure the thickness of the shear band in our computations and to observe that it is mesh independent provided the elements are small enough. In such local second gradient model, the width of the shear band is proportional to the parameter $D$ of the second gradient model [24].

Observation of the convergence profile obtained through Newton-Raphson iterations in the previous modelling validates the analytical expressions of the element stiffness matrix detailed in Section 6. Figure 5 shows a rapid decrease of the error norm up to the numerical noise. The different curves correspond to steps at a given value of axial strain (EPS). Eventually, these 

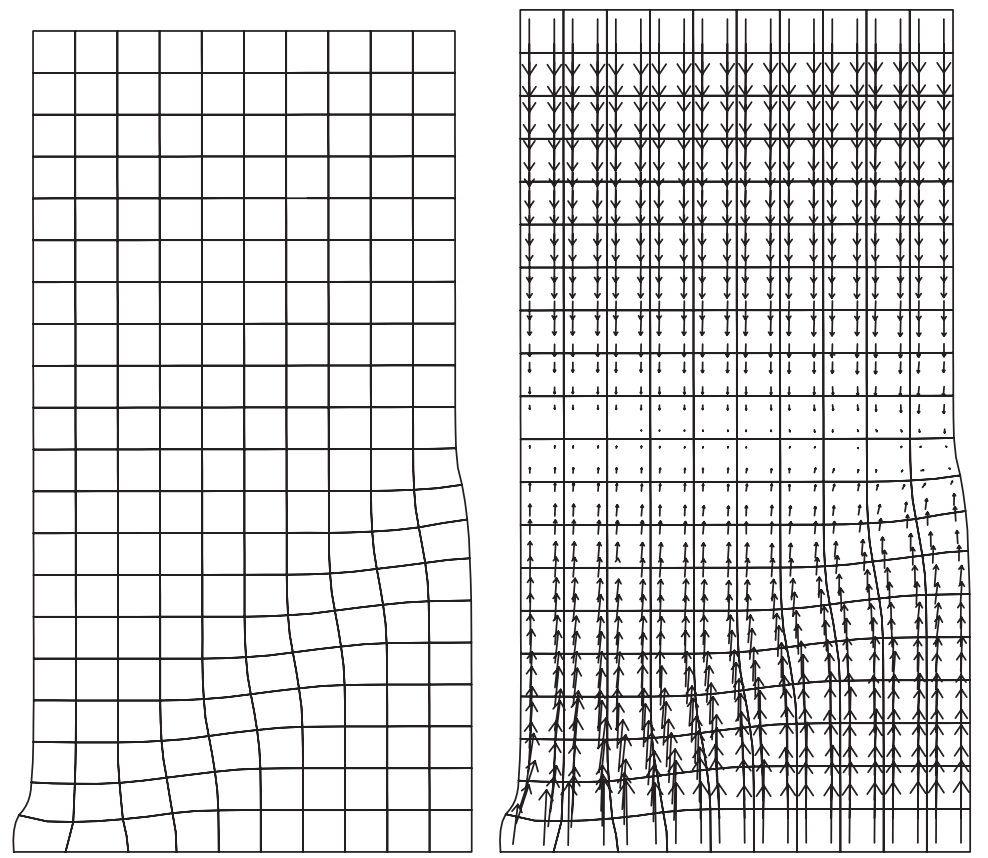

Figure 3. Localization mode and water flow after $2.5 \%$ axial strain $\left(\kappa=10^{-12} \mathrm{~m}^{2}\right)$.

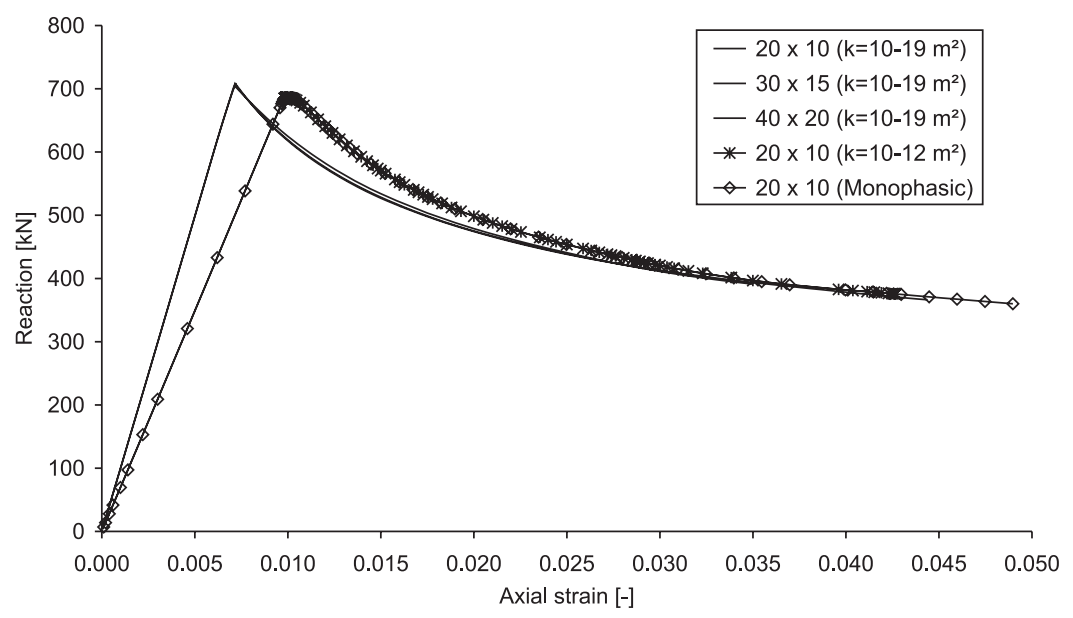

Figure 4. Axial strain vs global reaction for five biaxial tests.

preliminary computations show that the permeability has an influence on the loading curve (Figure 4) but not on the shear band thickness (Figures 7 and 8). This observation is of course only valid for the model used in the computation. 


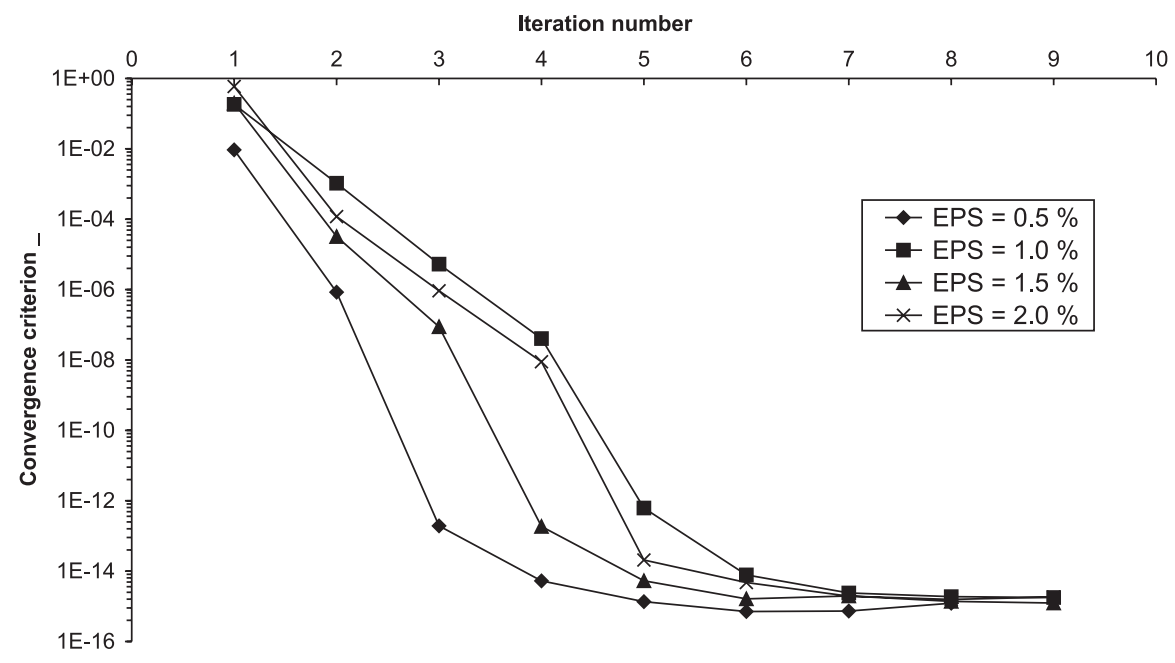

Figure 5. Convergence profile in coupled biaxial test.
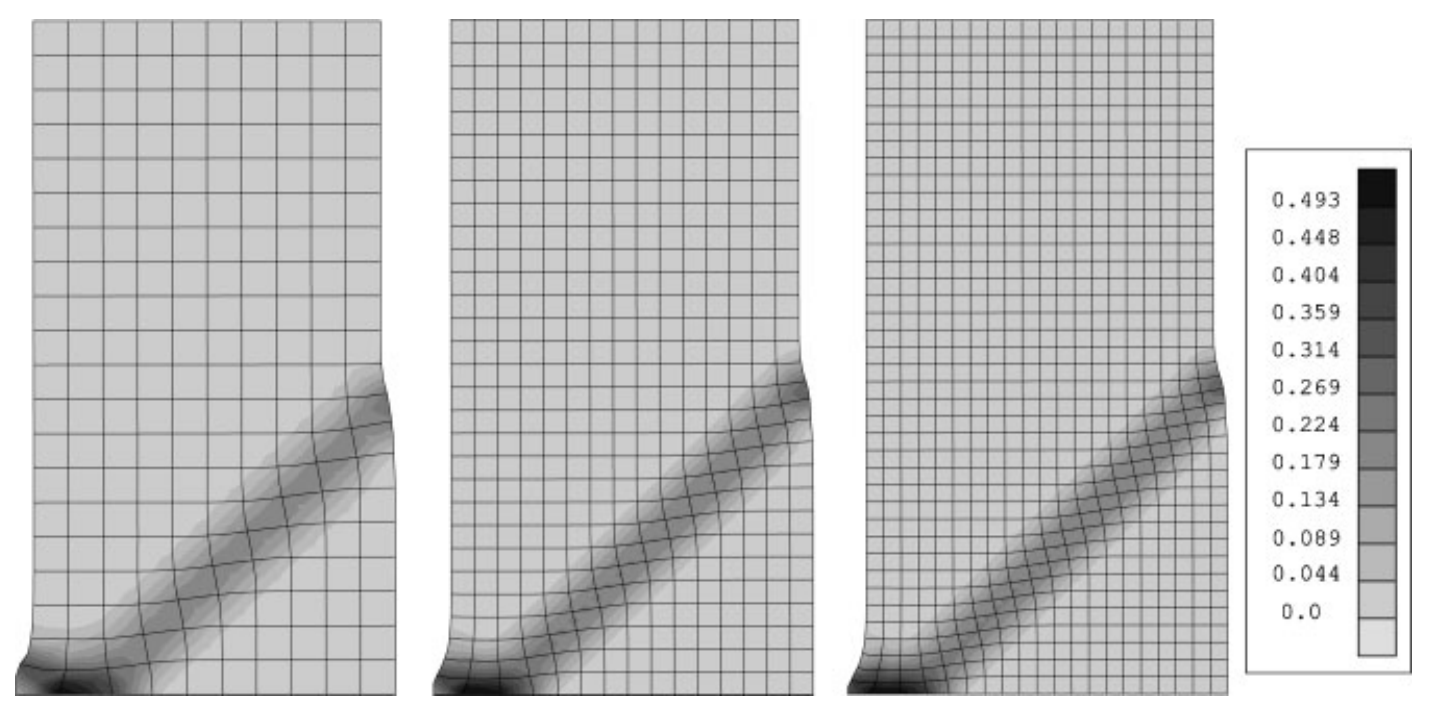

Figure 6. Deviatoric deformation after $2.5 \%$ axial strain $\left(\kappa=10^{-19} \mathrm{~m}^{2}\right)$.

\section{CONCLUSIONS}

Enhanced models are necessary to obtain regularized solutions for localized plastic strain fields computed with a finite element method. Many enhancements have been developed in the past, especially in the case of monophasic materials. However, geomaterials are mainly porous media 

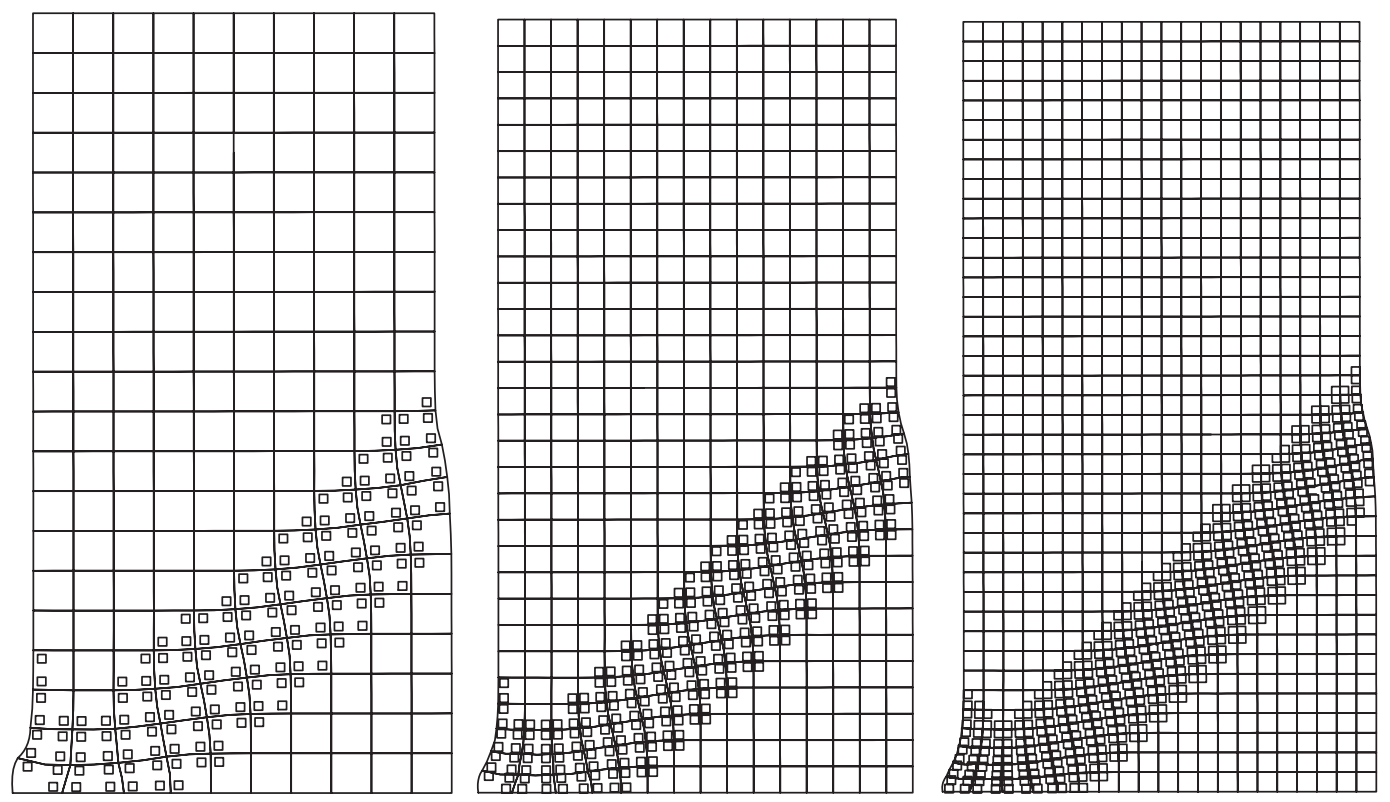

Figure 7. Plastic zones after $2.5 \%$ axial strain $\left(\kappa=10^{-19} \mathrm{~m}^{2}\right)$.
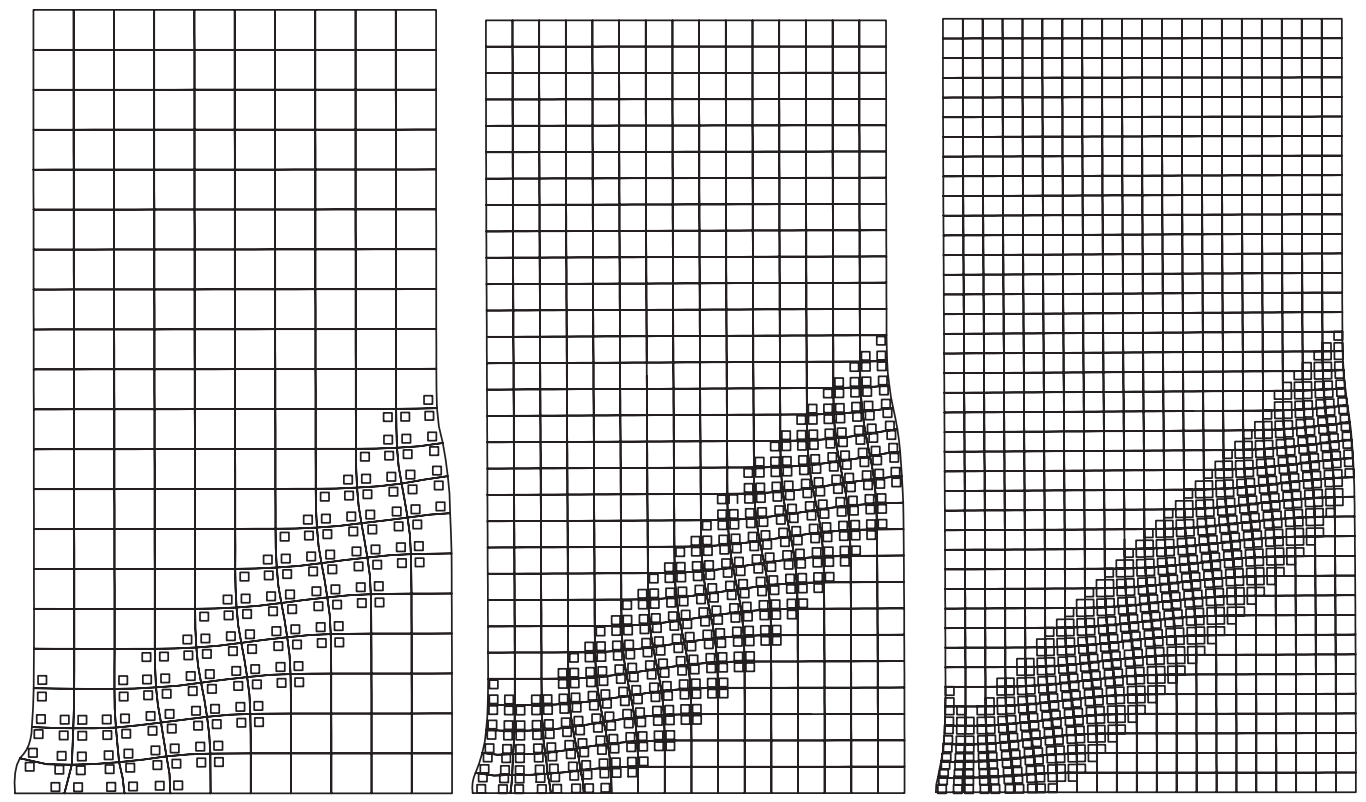

Figure 8. Plastic zones after $2.5 \%$ axial strain $\left(\kappa=10^{-19} \mathrm{~m}^{2}\right)$. 
filled by one or more fluids and it is thus interesting to study the influence of the fluid on the localization problem.

In this paper, our analysis is restricted to saturated soils or rocks filled by one compressible pore fluid. A coupled second gradient formulation has been proposed in the framework of microstructure continuum. A complete description of a large strain finite element using this local second gradient coupled model has been presented and first numerical results have been obtained for biaxial tests modelling.

Numerical results show clearly the regularization of the localization pattern. This study is only a preliminary one and some further investigations are necessary in order to draw more clear conclusions on the influence of permeability. Indeed the numerical effort for local second gradient modelling is essentially concerned with the development of the finite element. In the next future, more realistic constitutive models (non-associativity, yield function depending on mean stress, etc...) will be directly used in coupled applications.

\section{ACKNOWLEDGEMENTS}

The results presented in this paper have been obtained during a first post doctoral stay in Laboratoires 3S-Grenoble with a financial support of the European project RTN-DIGA (Contract No. HPRN-CT-2002-00220) and a second post doctoral stay granted by Fonds National de la Recherche Scientifique of Belgium. They are both gratefully acknowledged for their supports.

\section{REFERENCES}

1. Bowen RM. Incompressible porous media models by use of the theory of mixtures. International Journal of Engineering Science 1980; 18:1129-1148.

2. de Boer R. Theory of Porous Media. Springer: Berlin, 2000.

3. Hassanizadeh M, Gray W. General conservation equations for multi-phase systems: 1. Average procedure. Advances in Water Resources 1979; 2:131-144.

4. Hassanizadeh M, Gray W. General conservation equations for multi-phase systems: 2. Mass, momenta, energy, and entropy equations. Advances in Water Resources 1979; 2:191-208.

5. Lewis RW, Schrefler BA. The Finite Element Method in the Static and Dynamic Deformation and Consolidation of Porous Media. Wiley: New York, 2000.

6. Biot MA. General theory of three-dimensional consolidation. Journal of Applied Physics 1941; 12:155-164.

7. Coussy O. Mechanics of Porous Continua. Wiley: London, 1995.

8. Truesdell C, Noll W. The non-linear fields theory of mechanics. Encyclopedia of Physics. Springer: Berlin, 1965.

9. Desrues J. La localisation de la déformation dans les matériaux granulaires. Thèse de Doctorat es Sciences, 1984.

10. Desrues J, Viggiani G. Strain localization in sand: an overview of the experimental results obtained in grenoble using stereophotogrammetry. International Journal for Numerical and Analytical Methods in Geomechanics 2004; 28(4):279-321.

11. Bésuelle P, Desrues J, Raynaud S. Experimental characterization of the localization phenomenon inside a Vosges sandstone in a triaxial cell. International Journal of Rock Mechanics and Mining Sciences 2000; 37:1223-1237.

12. Baud P, Klein E, Ten-Fong W. Compaction localization in porous sandstone: evolution of damage and acoustic emission activity. Journal of Structural Geology 2004; 26:603-624.

13. Pijaudier-Cabot G, Bažant ZP. Nonlocal damage theory. Journal of Engineering Mechanics (ASCE) 1987; 113:1512-1533.

14. Aifantis EC. On the microstructural origin of certain inelastic models. Journal of Engineering Materials and Technology (ASME) 1984; 106:326-330. 
15. Vardoulakis I, Sulem J. Bifurcation Analysis in Geomechanics. Blackie Academic and Professional: London, 1995.

16. Bazant ZP, Belytschko TB, Chang TP. Continuum model for strain softening. Journal of Engineering Mechanics (ASCE) 1984; 110:1666-1692.

17. de Borst R, Mühlhaus H. Gradient dependant plasticity: formulation and algorithmic aspects. International Journal for Numerical Methods in Engineering 1992; 35:521-539.

18. Cosserat E, Cosserat F. Théorie des corps déformables. A Hermann et Fils: Paris, 1909.

19. Mühlhaus H, Vardoulakis I. The thickness of shear bands in granular materials. Géotechnique 1987; 37: 271-283.

20. Germain P. La méthode des puissances virtuelles en mécanique des milieux continus: Première partie: théorie du second gradient. Journal de Mécanique 1973; 12(2):235-274.

21. Germain P. The method of virtual power in continuum mechanics part 2: microstructure. SIAM Journal on Applied Mathematics 1973; 25(3):556-575.

22. Mindlin R. Second gradient of strain and surface-tension in linear elasticity. International Journal of Solids and Structures 1965; 1:417-438.

23. Mindlin RD, Eshel NN. On first strain gradient theories in linear elasticity. International Journal of Solids and Structures 1968; 4:109-124.

24. Chambon R, Caillerie D, ElHassan N. One-dimensional localization studied with a second grade model. European Journal of Mechanics - A/Solids 1998; 17:637-656.

25. Chambon R, Caillerie D, Matsushima T. Plastic continuum with microstructure, local second gradient theories for geomaterials: localization studies. International Journal of Solids and Structures 2001; 38:8503-8527.

26. Chambon R, Caillerie D, Tamagnini C. A strain gradient plasticity theory for finite strain. Computer Methods in Applied Mechanics and Engineering 2004; 193:2797-2826.

27. Ehlers W, Volk W. On theoretical and numerical methods in the theory of porous media based on polar and non-polar elasto-plastic solid materials. International Journal of Solids and Structures 1998; 35:4597-4617.

28. Ehlers W, Ellsiepen P, Ammann M. Time- and space-adaptive methods applied to localization phenomena in empty and saturated micropolar and standard porous media. International Journal for Numerical Methods in Engineering 2001; 52:503-526.

29. Zhang HW, Schrefler BA. Gradient-dependent plasticity model and dynamic strain localization analysis of saturated and partially saturated porous media: one dimensional model. European Journal of Mechanics A/Solids 2000; 19:503-524.

30. Zhang C, Schrefler BA. Particular aspects of internal length scales in strain localization analysis of multiphase porous materials. Computer Methods in Applied Mechanics and Engineering 2004; 193:2867-2884.

31. Benallal A, Comi C. Perturbation growth and localisation in fluid-saturated inelastic porous media under quasi-static loadings. Journal of the Mechanics and Physics of Solids 2003; 51:851-899.

32. Borja R, Alarcon E. A mathematical framework for finite strain elastoplastic consolidation part 1: balance law, variational formulation and linearization. Computers Methods in Applied Mechanics and Engineering 1995; 122:765-781.

33. Zienkiewicz O, Taylor R. The Finite Element Method (5th edn). Butterworth-Heinemann: Stonchem, MA, 2000.

34. Collin F. Couplages thermo-hydro-mécaniques dans les sols et les roches tendres partiellement saturés. Thèse de Doctorat, Université de Liège (231), 2003; 300.

35. Matsushima T, Chambon R, Caillerie D. Large strain finite element analysis of a local second gradient model: application to localization. International Journal for Numerical Methods in Engineering 2002; 54:499-521.

36. Chambon R, Moullet JC. Uniqueness studies in boundary value problems involving some second gradient models. Computer Methods in Applied Mechanics and Engineering 2004; 193:2771-2796.

37. Fleck N, Hutchinson J. Strain gradient plasticity. Advances in Applied Mechanics 1997; 33:295-361.

38. Simo JC, Taylor RL. Consistent tangent operators for rate-independent elastoplasticity. Computer Methods in Applied Mechanics and Engineering 1985; 48:101-118.

39. Felippa C, Park K. Staggered transient analysis procedures for coupled mechanical systems: formulation. Computer Methods in Applied Mechanics and Engineering 1980; 24:61-111.

40. Park K, Felippa C. Partitioned transient analysis procedures for coupled field problems: accuracy analysis. Journal of Applied Mechanics 1980; 47:919-926. 
41. Charlier R, Radu JP, Barnichon JD. Water movement effect on the strain localisation during a biaxial compression. In NUMOG VI-Seventh International Symposium on Numerical Models in Geomechanics, Pande G, Pietruszczak S (eds). Balkema: Rotterdam, 1997; 219-224.

42. Zhang C, Sanavia L, Schrefler BA. Numerical analysis of dynamic strain localisation in initially saturated dense sand with a modified generalised plasticity model. Computers and Structures 2001; 79:441-459.

43. Chambon R, Crochepeyre S, Charlier R. An algorithm and a method to search bifurcation points in non-linear problems. International Journal for Numerical Methods in Engineering 2001; 51:315-332. 